\title{
Intra-Arterial Delivery of Idarubicin in Two Patients with Glioblastoma
}

\author{
Mohamad Chehimi $^{\mathrm{a}} \quad$ Mathieu Boone $^{\mathrm{a}}$ Cyril Chivot $^{\mathrm{b}}$ Hervé Deramond $^{\mathrm{b}}$ \\ Jean-Marc Constans ${ }^{b}$ Mony Chenda Ly ${ }^{a}$ Bruno Chauffert ${ }^{a}$ \\ ${ }^{a}$ Department of Medical Oncology, University Hospital, Amiens, France; ${ }^{b}$ Department of \\ Radiology, University Hospital, Amiens, France
}

\section{Keywords}

Glioblastoma · Idarubicin · Intra-arterial chemotherapy

\begin{abstract}
There is no effective treatment for recurrent glioblastoma (GB) when temozolomide-based radiochemotherapy fails. In theory, intra-arterial (IA) delivery of cytotoxic agents could achieve higher drug concentrations in tumors compared to intravenous injection. Moreover, choosing a highly lipid-soluble drug could make the most of the first-pass effect. Here, we evaluated idarubicin (IDA), a lipophilic anthracycline, in an in vitro assay using four human GB cell lines and compared it with 11 other drugs previously used for the IA treatment of brain tumors. Despite impressive in vitro cytotoxicity, IA IDA did not produce a beneficial effect in 2 patients with recurrent GB.

(C) 2016 The Author(s)

Published by S. Karger AG, Basel
\end{abstract}

\section{Introduction}

No effective treatment exists against recurrent glioblastoma (GB) when the standard first-line treatment using a temozolomide-based radiochemotherapy fails. Bevacizumab and lomustine increased progression-free survival, but their impact on overall survival is controversial [1]. There is no defined third-line treatment. Intra-arterial (IA) delivery of cytotoxic drugs has been explored over decades, but with no significant results to date [for review, see 2]. IA studies commonly use the same drugs as for i.v. delivery (melphalan, carboplatin, 
methotrexate, and temozolomide). Drugs are not specifically selected in order to exploit the concentration/antitumor effect anticipated by using IA delivery. Drugs have to be rapidly and irreversibly taken up during their first pass through the tissue circulation [3]. Moreover, the selected drug should be highly cytotoxic against targeted tumor cells. Here, we evaluated the in vitro cytotoxicity of idarubicin (IDA), a lipophilic anthracycline, using four human GB cell lines. Encouraging in vitro data led us to evaluate IDA by the IA route in patients with recurrent GB.

\section{Results}

In vitro Results

IDA cytotoxicity was compared to 11 other antineoplastic drugs previously used for IA chemotherapy in GB or brain metastases in 4 human GB cell lines (fig. 1). Briefly, U87MG, CGL-1 CGL-3 or CGL-8 cells were seeded in complete culture medium and grown for $48 \mathrm{~h}$ in cell-culture plates. U87-MG cells were obtained from American Type Culture Collection (Manassas, Va., USA). The CGL-1, CGL-3 and CGL-9 cell lines were established from resected GB from 3 different patients and kindly donated by Dr. Philipe Genne (Oncodesign, Dijon, France). Subconfluent cells were incubated for $30 \mathrm{~min}$ with antitumor drugs at one selected concentration, then washed and grown again for 7 days in drug-free culture medium. The selected drug concentration was calculated assuming an unilateral carotid blood flow of 0.33 liter/min (10 liters in $30 \mathrm{~min}$ ) and taking into account the dose usually given i.v. in patients with various tumors (table 1). The 30-min duration of in vitro exposure was selected since it was tolerated by patients receiving intracarotid infusion. A colorimetric assay was used to quantify drug-induced cell death. Surviving cells adherent to the bottom of the well were fixed with pure ethanol and stained with crystal violet dye. The dye was eluted with 33\% acetic acid. Absorbance was determined by spectrophotometry (UVM 340; Bioserv) at 570 $\mathrm{nm}$ wavelength. Percent cell survival was calculated as optical density in treated wells/ optical density in control wells $\times 100$.

Ninety percent or more of the cells from the 4 human GB cell lines were killed after a 30min exposure to $3 \mu \mathrm{g} / \mathrm{ml}$ IDA. This is an achievable concentration in the brain blood flow for a 30-mg dose of IDA. Other drugs were active less consistently.

\section{Case Presentations}

The impressive in vitro results led us to design a phase 1 study of IDA administration via intracarotid delivery (IDACAR) to treat recurrent GB. The IDACAR trial was approved on January 12, 2012, by the ethics committee (Comité de Protection des Personnes Nord-Ouest 2) and registered under EUDRACT No. 2011-004176-11. A dose increase was planned. After femoral puncture under local anesthesia, the catheter was placed into a carotid branch (S1, A1 or M 1, depending on the tumor localization) and above the ophthalmic artery in order to minimize ocular complications. Whole perfusion of the tumor was checked by angiography. IDA (1 $\mathrm{mg} / \mathrm{ml}$ in saline solution) was infused in the carotid blood flow by an electric pump over $30 \mathrm{~min}$. Patients were kept conscious and regularly asked to speak and move their limbs. 


\section{Patient 1}

A 56-year-old female had a history of resected colon adenocarcinoma in 2004. She was referred for behavioral disorders. A right frontal tumor with cystic contingent was evidenced by MRI. Partial resection of a GB was done in October 2010, followed by 60-Gy radiotherapy with concomitant and adjuvant temozolomide for 6 months. Disease recurred in November 2011 and progressed on MRI despite 3 months of bevacizumab. WHO status was 2 at inclusion with a mild left hemiparesis. The catheter was placed into the superficial branch of the middle cerebral artery that mainly irrigated the tumor. No adverse effects were observed during the IA injection of $22 \mathrm{mg}$ IDA $\left(12 \mathrm{mg} / \mathrm{m}^{2}\right)$ over $30 \mathrm{~min}$. Anisocoria, worsening of the left hemiparesis, and severe cognitive impairment occurred at day 3 after IA infusion. The neurological degradation was considered to be a case of irreversible and severe toxicity, although tumor progression could not be completely ruled out considering the MRI at day 28 (fig. 2). A grade III leuconeutropenia occurred at day 7 despite granulocyte colonystimulating factor (G-CSF). Grade I anemia and thrombocytopenia were also recorded. The patient died 42 days after IA IDA due to disease progression.

\section{Patient 2}

A 57-year-old female had an expansive sustentorial left occipital tumor. Complete resection of a GB in January 2009 was followed by 60-Gy radiotherapy with concomitant and adjuvant temozolomide for 6 months. Disease progressed in November 2011, and the patient received bevacizumab until March 2012. WHO status was 1 at inclusion. A catheter was placed into the posterior cerebral artery, and $21 \mathrm{mg}$ IDA were infused IA over $30 \mathrm{~min}$. No adverse effects occurred during the IA injection. Grade III leuconeutropenia was registered at day 7 nadir despite G-CSF. The neurological status and WHO status remained stable for 30 days. MRI evaluation on day 28 showed tumor stability according to the Response Assessment in Neuro-Oncology (RANO) Criteria. The patient refused a second IA injection and died 26 weeks after treatment due to disease progression.

Considering the possible grade IV neurological toxicity in the first patient and the lack of clinical benefit in both patients, the Independent Data Monitoring Committee (IDMC) recommended to stop the trial.

\section{Discussion}

We reported here for the first time the IA delivery of IDA in 2 patients with recurrent GB. Our in vitro results were in agreement with previous papers. Schott and Robert [4] compared growth inhibition, DNA synthesis inhibition, and cell incorporation of eight anthracyclines in a model of doxorubicin-sensitive and -resistant rat C6 GB cells. They found that new anthracyclines, including IDA, were more potent than the reference drugs (daunorubicin and doxorubicin) in rat GB cells. They observed reduced cross-resistance of IDA, pirarubicin and 4'-deoxy-4'-iododoxorubicin in a doxorubicin-resistant cell line. Among these anthracyclines, only IDA still remains available for clinical treatment of acute myeloid leukemias. Kuffel et al. [5] evaluated the growth-inhibitory and DNA-damaging activities of IDA, daunorubicin, doxorubicin, and epirubicin against human tumor cell lines, including the U87-MG human GB cell line. IDA was 2-5 times more potent than the other three anthracycline analogs. Considering that IDA is more lipophilic than other anthracyclines and more cytotoxic in glioma cell lines, Boogerd et al. [6] found a significant uptake of IDA and its major metabolite idarubicinol by the brain tumor tissue from 1 patient with a brain metastasis from breast 
cancer and from 4 patients with malignant glioma after a single oral dose of IDA (25-45 $\mathrm{mg} / \mathrm{m}^{2}$ ). Based on these preclinical data, a phase II trial was performed in children with progressive brain tumors [7]. i.v. IDA $\left(18 \mathrm{mg} / \mathrm{m}^{2}\right)$ was followed by G-CSF. Most patients developed progressive disease. Grade III/IV hematopoietic toxicities were common. The authors concluded that it is unlikely that IDA will be useful for the treatment of primary central nervous system tumors by the i.v. route.

In parallel to GB, we performed an experimental study to select the best candidate drug for transarterial chemoembolization of hepatocellular carcinoma (HCC). The SNU-398, HepG2, and SNU-449 human HCC cell lines were exposed to doxorubicin, epirubicin, IDA, mitoxantrone, carboplatin, cisplatin, oxaliplatin, 5-fluorouracil, gemcitabine, mitomycin C, or paclitaxel for $30 \mathrm{~min}$. IDA was the most active drug against all three HCC cell lines [8]. A phase I trial of IDA loaded on eluting beads for chemoembolization produced encouraging results in 21 patients with HCC and liver cirrhosis (response rate of $52 \%$ and $28 \%$ of complete responses) [9].

Considering the experimental data from our study and others about the strong in vitro antineoplasic activity of IDA and arguing that IA delivery could theoretically increase the tumor drug concentration by a factor of 5-10, we designed the IDACAR trial in recurrent GB. In contrast to HCC, clinical results were disappointing in our 2 GB patients. Neither of the 2 patients displayed a response on MRI, and irreversible grade IV neurological toxicity was suspected in the first patient. Many hypotheses can be proposed to explain the discrepancy between the in vitro and the clinical results. Selecting a drug that is potent on GB cells in vitro is not enough to improve the antitumor effect in patients. Heterogeneity of perfusion into the complex angioarchitecture of $\mathrm{GB}$, reduced drug penetration due to the residual blood-brain barrier, and drug streaming in the locally accelerated blood flow and a number of other technical and pharmacological pitfalls remain to be solved in order to improve the IA treatment of brain tumors [2]. Moreover, the lack of experimental models of GB in big animals greatly hinders progress.

\section{Acknowledgment}

This work was funded by an internal grant from the University Hospital of Amiens, France.

\section{Statement of Ethics}

The authors have no ethical conflicts to disclose.

\section{Disclosure Statement}

The authors have no conflicts of interest to declare. 


\section{References}

-1 Taal W, Oosterkamp HM, Walenkamp AM, Dubbink HJ, Beerepoot LV, Hanse MC, Buter J, Honkoop AH, Boerman D, de Vos FY, Dinjens WN, Enting RH, Taphoorn MJ, van den Berkmortel FW, Jansen RL, Brandsma D, Bromberg JE, van Heuvel I, Vernhout RM, van der Holt B, van den Bent MJ: Single-agent bevacizumab or lomustine versus a combination of bevacizumab plus lomustine in patients with recurrent glioblastoma (BELOB trial): a randomised controlled phase 2 trial. Lancet Oncol 2014;15:943-953.

-2 Ellis JA, Banu M, Hossain SS, Singh-Moon R, Lavine SD, Bruce JN, Joshi S: Reassessing the role of intraarterial drug delivery for glioblastoma multiforme treatment. J Drug Deliv 2015;2015:405735.

-3 Dedrick RL: Arterial drug infusion: pharmacokinetic problems and pitfalls. J Natl Cancer Inst 1988;80:84-89.

-4 Schott B, Robert J: Comparative cytotoxicity, DNA synthesis inhibition and drug incorporation of eight anthracyclines in a model of doxorubicin-sensitive and -resistant rat glioblastoma cells. Biochem Pharmacol 1989;38:167-172.

5 Kuffel MJ, Reid JM, Ames MM: Anthracyclines and their C-13 alcohol metabolites: growth inhibition and DNA damage following incubation with human tumor cells in culture. Cancer Chemother Pharmacol 1992;30:51-57.

6 Boogerd W, Tjahja IS, van de Sandt MM, Beijnen JH: Penetration of idarubicin into malignant brain tumor tissue. J Neurooncol 1999;44:65-69.

-7 Dreyer ZE, Kadota RP, Stewart CF, Friedman HS, Mahoney DH, Kun LE, McCluggage CW, Burger PC, Kepner J, Heideman RL; Pediatric Oncology Group: Phase 2 study of idarubicin in pediatric brain tumors: Pediatric Oncology Group study POG 9237. Neuro Oncol 2003;5:261-267.

-8 Boulin M, Guiu S, Chauffert B, Aho S, Cercueil JP, Ghiringhelli F, Krause D, Fagnoni P, Hillon P, Bedenne L, Guiu B: Screening of anticancer drugs for chemoembolization of hepatocellular carcinoma. Anticancer Drugs 2011;22:741-748.

9 Boulin M, Hillon P, Cercueil JP, Bonnetain F, Dabakuyo S, Minello A, Jouve JL, Lepage C, Bardou M, Wendremaire M, Guerard P, Denys A, Grandvuillemin A, Chauffert B, Bedenne L, Guiu B: Idarubicinloaded beads for chemoembolisation of hepatocellular carcinoma: results of the IDASPHERE phase I trial. Aliment Pharmacol Ther 2014;39:1301-1313. 


\section{Case Reports in Oncology}
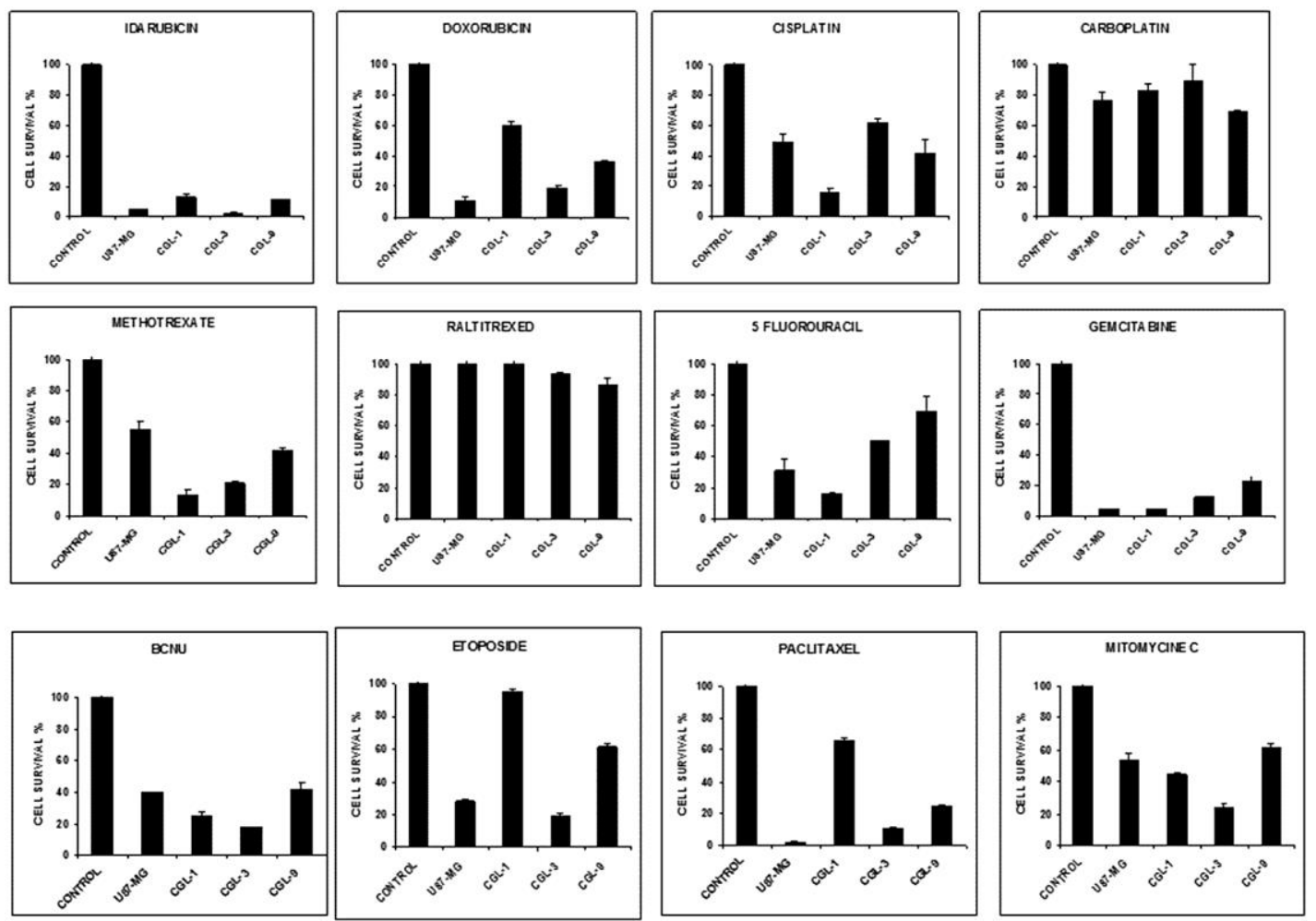

Fig. 1. In vitro colorimetric assay of 12 chemotherapeutic agents using four human GB cell lines. Bars indicate the mean of 3 wells (standard deviation $<10 \%$ ).

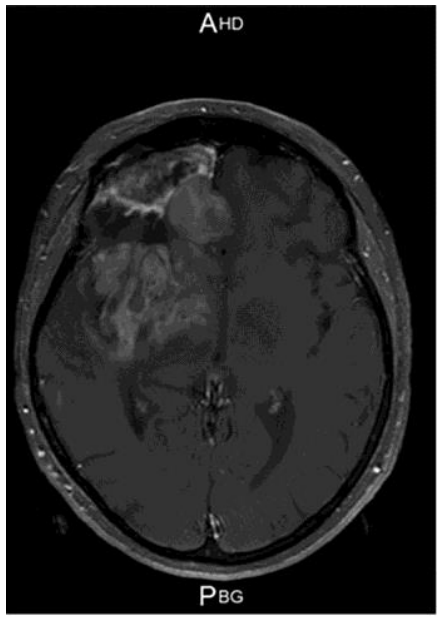

Before

IA treatment

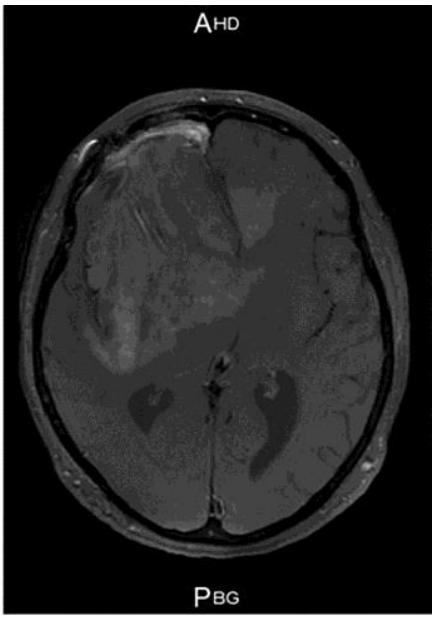

Day 28 after

IA treatment

Fig. 2. Gadolinium-enhanced T1-weighted MRI before and 28 days after the IA delivery of IDA in Patient 1. 
Table 1. Calculations of drug concentrations for the in vitro cytotoxicity assay

\begin{tabular}{lll}
\hline Drug & $\begin{array}{l}\text { Dose for i.v. } \\
\text { use, mg }\end{array}$ & $\begin{array}{l}\text { Concentration for } \\
\text { the in vitro assay, mg/l }\end{array}$ \\
\hline Idarubicin & 30 & 3 \\
Doxorubicin & 60 & 6 \\
Cisplatin & 150 & 15 \\
Carboplatin & 800 & 80 \\
Methotrexate & 100 & 10 \\
Raltitrexed & 6 & 0.6 \\
5-fluorouracil & 1,000 & 100 \\
Gemcitabine & 2,000 & 200 \\
BCNU (carmustine) & 200 & 20 \\
Etoposide & 300 & 30 \\
Paclitaxel & 300 & 30 \\
Mitomycin C & 30 & 3 \\
\hline
\end{tabular}

Blood flow in the selected carotid artery was estimated to be at 0.33 $\mathrm{ml} / \mathrm{min}$ (10 liters in $30 \mathrm{~min}$ ). The selected drug doses were in the range of those used by i.v. route for various tumors. 